\title{
RUIN PROBABILITIES AND DEFICIT FOR THE RENEWAL RISK MODEL WITH PHASE-TYPE INTERARRIVAL TIMES
}

\author{
BY
}

\author{
F. AVRAM AND M. UsÁBEL
}

\begin{abstract}
This paper shows how the multivariate finite time ruin probability function, in a phase-type environment, inherits the phase-type structure and can be efficiently approximated with only one Laplace transform inversion.

From a theoretical point of view, we also provide below a generalization of Thorin's formula (1971) for the double Laplace transform of the finite time ruin probability, by considering also the deficit at ruin; the model is that of a Sparre Andersen (renewal) risk process with phase-type interarrival times.

In the case when the claims distribution is of phase-type as well, we obtain also an alternative formula for the single Laplace transform in time (or "exponentially killed probability"), in terms of the roots with positive real part of the Lundberg's equations, which complements Asmussen's representation (1992) in terms of the roots with negative real part ${ }^{1}$.
\end{abstract}

\section{KEYWORDS}

Finite time ruin probability, exponentially killed ruin probability, deficit at ruin, Sparre Andersen Model, phase-type distributions.

\section{INTRODUCTION}

The convenience of phase-type modelling has been amply demonstrated in both risk and queueing theory, but mostly for ultimate ruin probabilities. For the considerably more challenging case of finite time ruin probabilities with deficit at ruin, phase-type approximations were recently provided only in the classical Poisson arrivals case in Asmussen, Avram and Usábel (2001) (using Erlang killing) and in Avram and Usábel (2001) (via a single Laplace inversion).

This work is continued below; under the assumption of phase-type interarrival times, we extend first the formula of the double Laplace transform of the finite time ruin probability of a renewal risk process, obtained by Thorin

1 The authors gratefully acknowledge the financial support from the Dep. of Actuarial Mathematics and Statistics, Heriot-Watt University, Edinburgh and MCyT SEC2001-1169. 
(1971) and by Cohen (1985) in the context of queueing theory, to include the deficit at ruin as well. Our solution emphasizes the importance of a certain "structure matrix" (see below), already encountered in the previously studied classic case with phase-type claims.

We consider a Sparre Andersen risk process in continuous time $\left\{Z_{t}\right\}_{t \geq 0}$ with $U_{k}$ claim sizes and premium $c$ per time unit, i.e.

$$
Z_{t}=u+c t-\sum_{k=1}^{N_{t}} U_{k}
$$

where $u$ are the initial reserves and $N_{t}$ the total number of claims up to time $t$. The claim times sequence forms an ordinary renewal process with common c.d.f. of the waiting times between claims $A(t)$ with mean $1 / \lambda$. The claims $\left\{U_{k}\right\}$ are independent one another and also independent of $N_{t}$, with c.d.f. $B(u)$ the distribution function of claim sizes $U_{k}$ with mean $\mu$. The premium rate is usually defined in actuarial literature as $c=\lambda \mu(1+\theta)$ where $\theta$ is the relative safety loading. The distributions $A(t), B(u)$ are concentrated on $(0, \infty)$ and have densities and Laplace transforms denoted by $a(t), b(u)$ and $a^{*}(m), b^{*}(n)$, respectively. We consider here the case when furthermore $A(t)$ and sometimes $B(u)$ are of "phase-type" (see below).

Let us now define $\tau=\inf \left\{w>0: Z_{w}<0\right\}$ as the ruin time and $Y=-Z_{\tau}$ as the deficit exactly at ruin time or severity of ruin. Let

$$
\Psi_{t, u, y}=P_{u}\{\tau<t, Y \leq y\}
$$

denote the probability of ruin within time span $t$, with initial reserves $u$ and severity of ruin less than $y$ (the particular case $\Psi_{u, v}=P_{u}\{\tau<\infty, Y \leq y\}$ being known as the perpetual or ultimate ruin probability).

For a particular choice of the parameters $u$ and $y$, the function $\Psi_{t, u, v}$ is a defective distribution function with respect $t$; we study below the density function of the ruin time

$$
\psi_{t, u, y}=\frac{\partial \Psi_{t, u, y}}{\partial t}
$$

This interesting function has been treated frequently in the actuarial literature; see the related works by Egidio dos Reis (1993, 2000), Dickson and Egidio dos Reis (1996) and Willmot (1998, 2000).

Using a renewal argument, see for instance Thorin $(70,71,82)$, we can easily obtain the following integral equation for the multivariate ruin probability (with deficit at ruin $y$ ) for the ordinary case

$$
\begin{aligned}
\Psi_{t, u, y} & =\int_{0}^{t}(B(u+c s+y)-B(u+c s)) a(s) d s \\
& +\int_{0}^{t} \int_{0}^{u+c s} \Psi_{t-s, u+c s-z, y} b(z) a(s) d z d s
\end{aligned}
$$


The corresponding equation for the density is

$$
\begin{aligned}
\psi_{t, u, y} & =(B(u+c t+y)-B(u+c t)) a(t) \\
& +\int_{0}^{t} \int_{0}^{u+c s} \psi_{t-s, u+c s-z, y} b(z) a(s) d z d s
\end{aligned}
$$

The classical approach to solve the ruin problem, going back to Cràmer (1955) and Sparre Andersen (1955), consists in applying integral transforms to the former integral equations, like for instance the double Laplace-Stieltjes operator

$$
\Psi_{m, n, y}^{* *}=\int_{t=0}^{\infty} \int_{u=0}^{\infty} \Psi_{t, u, y} e^{-m t-n u} d u d t
$$

to the integral equations above. Then the original ruin probability function can be approximated using Laplace transform inversion techniques, see Usábel $(1999,2001)$ and Thorin $(1970,71,73,77,82)$, Thorin and Wikstad (1973), Wikstad $(1971,77)$ who used the Piessens $(1969)$ inversion method of the Laplace transform, Bohman $(1971,74,75)$ who used the Fourier transform and Seal $(1971,74,77)$ who dealt with both Laplace and Fourier numerical inversions.

Assumption: We assume throughout the paper that the interarrival distribution is $\operatorname{PH}(\alpha, \boldsymbol{A}, r)$, i.e. that

$$
A(t)=1-\alpha e^{t} \boldsymbol{A}_{1}
$$

where $\mathbf{A}$ is a Markovian subgenerator matrix of order $r$ (i.e. with all offdiagonal elements nonnegative, and such that the vector $\mathbf{a}=-\mathbf{A} \mathbf{1}$ has nonegative elements, out of which at least one is strictly positive). It follows that the density is:

$$
a(t)=\alpha e^{\mathbf{A} t} \mathbf{a}
$$

The Laplace transform, defined by the integral $\int_{0}^{\infty} e^{-s t} A(d t)$ for $\operatorname{Re}(s) \geq 0$, is:

$$
a^{*}(s)=\alpha(s \mathbf{I}-\mathbf{A})^{-1} \mathbf{a}=\alpha a^{*}[s]
$$

and this extends for all $s \in \mathbb{C}$ except for the poles that are the eigenvalues of $\mathbf{A}$, by analytic continuation. An important role below will be played by the vector $\boldsymbol{a}^{*}[s]=(s \mathbf{I}-\mathbf{A})^{-1} \mathbf{a}$ of Laplace transforms with fixed starting phase.

We provide in the Appendix B some further necessary background on phasetype distribution; the reader is referred to Rolski, Schmidli, Schmidt and Teugels (1999) and Asmussen (2000) for other results and applications of this family.

Contents: In the present work, in section 2, we obtain in Theorem 1 the double Laplace transform for the multivariate problem (considering the deficit at ruin $y$ ) under the assumption of phase-type interarrivals, generalizing formula 3.4 of Thorin (1971). 
In section 3 an interesting application of theorem 1 is presented when the claim sizes are also of phase-type nature. This result, expressed in terms of the non-negative roots (not necessarily distinct) of the Lundberg's equation generalizes several particular cases which have been presented in the literature like Dickson and Hipp $(1998,2000)$ and Willmot (1999) and yields an alternative representation of Asmussen's result (1992) which had used the negative (assumed distinct) roots.

The numerical illustrations contained in section 4 clearly show that the method presented can lead to very efficient approximations of the rather challenging multivariate finite time ruin probabilities. Moreover, the approximations inherit the phase-type nature as well, see (15).

Finally, in section 5, a short hint on the applications to actuarial practice is offered, mainly based on the interesting properties of the phase-type distributions.

\section{The Double LAplaCe transform OF THE Finite TIME MULTIVARIATE RUIN PROBABILITY}

The following result yields the double Laplace transform of the multivariate ruin probabilities, including in the usually ignored case when the Cramer-Lundberg equation has multiple roots. We will denote by $f^{k}(n)$ the $k^{\prime}$ th derivative with respect to $n$ of a general function $f(n)$.

Theorem 1. Suppose that the waiting times of a risk process are distributed PH $(\alpha, \mathbf{A}, r)$ (assumed minimal PH representation). Let

$$
B_{y}^{*}(n)=\int_{a=0}^{\infty}(B(a+y)-B(a)) e^{-n a} d a
$$

and introduce the $r \times r$ structure matrix:

$$
\begin{aligned}
& \mathbf{C}_{m} \\
= & \left(\mathbf{a}^{* 0)}\left[m-c n_{m 1}\right], \ldots, \mathbf{a}^{\left.* \sigma_{m 1}-1\right)}\left[m-c n_{m 1}\right], \ldots, \mathbf{a}^{* 0)}\left[m-c n_{m q}\right], \ldots, \mathbf{a}^{\left.* \sigma_{m q}-1\right)}\left[m-c n_{m q}\right]\right)
\end{aligned}
$$

where $\left\{n_{m j}\right\}_{j=1}^{q}$ the complex roots with non-negative real part of the Cramer-Lundberg equation

$$
1-\mathrm{b}^{*}(n) a^{*}(m-c n)=0
$$

with respective multiplicities $\sigma_{m j}, j=1, \ldots, q$.

Then, the double Laplace transform of $\psi_{t, u, y}($ see (2)) is given for all complex $m$ and $n$ with non-negative real part by:

$\psi_{m, n, y}^{* *}=\int_{0}^{\infty} \int_{0}^{\infty} e^{-m t-n u} \psi_{t, u, y} d u d t=\frac{B_{y}^{*}(n) a^{*}(m-c n)-\mathbf{D}_{m, y} \mathbf{C}_{m}^{-1} \mathbf{a}^{*}[m-c n]}{1-b^{*}(n) a^{*}(m-c n)}$ 
where the row $1 \times r$ vector $\mathbf{D}_{m, y}$ is

$$
\mathbf{D}_{m, y}=\left(D_{m, y}^{0)}\left(n_{m 1}\right), \ldots, D_{m, y}^{\sigma_{m 1}-1}\left(n_{m 1}\right), \ldots, D_{m, y}^{0)}\left(n_{m q}\right), \ldots, D_{m, y}^{\sigma_{m q}-1}\left(n_{m q}\right)\right)
$$

and for simplicity in the notation $D_{m, y}(n)=B_{y}^{*}(n) a^{*}(m-c n)$

Proof. Applying the double-Laplace transform operator

$$
\psi_{m, n, y}^{* *}=\int_{0}^{\infty} \int_{0}^{\infty} e^{-m t-n u} \psi_{t, u, y} d u d t
$$

to (2) and using the lemma in Appendix A we find that

$$
\begin{aligned}
\psi_{m, n, y}^{* *} & =\psi_{m, n, y}^{* *} b^{*}(n) a^{*}(m-c n)+B_{y}^{*}(n) a^{*}(m-c n)-\mathbf{U}_{y}(m) \mathbf{a}^{*}[m-c n] \\
& =\frac{B_{y}^{*}(n) a^{*}(m-c n)-\mathbf{U}_{y}(m) \mathbf{a}^{*}[m-c n]}{1-b^{*}(n) a^{*}(m-c n)}
\end{aligned}
$$

where, for convenience in the notation

$$
\begin{aligned}
\mathbf{U}_{y}(m) & =\mathbf{G}_{y}(m)+\mathbf{H}_{y}(m) \\
& =\alpha\left(\psi_{m, \frac{m \mathbf{I}-\mathbf{A}}{c}, y}^{* *} b^{*}\left(\frac{m \mathbf{I}-\mathbf{A}}{c}\right)+B_{y}^{*}\left(\frac{m \mathbf{I}-\mathbf{A}}{c}\right)\right) \\
D_{m, y}(n) & =B_{y}^{*}(n) a^{*}(m-c n)
\end{aligned}
$$

and $\alpha^{*}[m-c n]=((m-c n) \mathbf{I}-\mathbf{A})^{-1} \mathbf{a}$ by definition.

To obtain the values of the row $1 \times r$ vector $\mathbf{U}_{y}(m)$ we use the fact that as a double Laplace transform, the function $\psi_{m, n, y}^{* *}$ is finite for any value of $n$, $\operatorname{Re}(n) \geq 0$, and hence that at $n_{m j}, j=1, \ldots, q$ the numerator of (6) must have roots of at least the same multiplicity as those of the denominator. The C-L equation has exactly $r$ roots ( $r$ is the order of the minimal $\mathrm{PH}$ representation) with non-negative real part. Something easy to prove bearing in mind that the Laplace transform $a^{*}(m-c n)$ is just a quotient of polynomials with denominator of order $r$ (greater than the one of the numerator).

This yields the following system of $r$ equations and unknowns

$$
\mathbf{U}_{y}(m) \mathbf{a}^{* l)}\left[m-c n_{m j}\right]=D_{m, y}^{l)}\left(n_{m j}\right) ; \quad j=1, \ldots, q \quad l=0, \ldots, \sigma_{m j}-1
$$

where $\mathbf{a}^{* l)}\left[m-c n_{m j}\right]$ and $D_{m, y}^{l)}\left(n_{m j}\right)$ are the $l$-th derivatives with respect $n$ at $n_{m j}$, or, using matrix notation

$$
\mathbf{U}_{y}(m) \mathbf{C}_{m}=\mathbf{D}_{m, y}
$$

The former system of equations has a unique solution because of the nature of $\mathbf{U}_{y}(m)$ (see (7)): indeed for a particular choice of $\alpha$ and $\mathbf{A}$, in this case the minimal 
PH representation, the values of the matrix functions $\psi_{m, \frac{m \mathrm{I}-\mathrm{A}}{c}, y}^{*}, b^{*}\left(\frac{m \mathbf{I}-\mathrm{A}}{c}\right)$ and $B_{y}^{*}\left(\frac{m \mathbf{I}-\mathrm{A}}{c}\right)$ are unique. This last fact means that should $\mathbf{C}_{m}$ be singular the former systems of equations had infinite solutions $\mathbf{U}_{y}(m)$ reaching an absurd statement. Subsequently

$$
\mathbf{U}_{y}(m)=\mathbf{D}_{m, y} \mathbf{C}_{m}^{-1}
$$

Note: This result reduces the ruin problem to the computation of the roots with nonnegative part of the Cramèr-Lundberg equation and that of $\mathbf{C}_{m}^{-1}$. Theorem 1 is an extension of the multivariate problem (considering the deficit at ruin $y$ ), under the assumption of phase-type interarrivals, generalizing formula 3.4 of Thorin(1971).

Unfortunately, using theorem 1, approximations to $\Psi_{t, u, v}$, considering a general claim size distribution $b(x)$, can only be obtained after two Laplace transform inversions. However, two interesting cases only need one Laplace transform inversion:

1. the ultimate multivariate ruin probability for general claim size distribution $b(x)$, easily obtained using just $\psi_{0, n, y}^{* *}$.

2. the finite time multivariate ruin probability for phase-type claim sizes, considered in the next section.

\section{Multivariate RUIN PROBABILITIES FOR PHASE-TYPE CLAIM SIZES AND WAITING TIMES}

Let us now focus on the phase-type environment both for claim sizes and waiting times. This particular choice is not too restrictive, because this family is dense in the sense that any probability distribution can be approximated using phase-type distributions (see the concluding comments section for details).

The following theorem offers the finite time multivariate ruin probability using only one Laplace inversion.

Theorem 2. Under the assumption of $P H(\beta, \mathbf{B}, k)$, claim sizes and $P H(\alpha, \mathbf{A}, r)$, waiting times are phase-type, the single Laplace transform in time of the multivariate ruin probability is

$$
\begin{aligned}
\Psi_{m, u, y}^{*} & =\left(\frac{1}{m}\right) \boldsymbol{\eta}_{m} e^{\mathbf{Q}_{m} u}\left(\mathbf{I}-e^{\mathbf{B} y}\right) \mathbf{1} \\
\mathbf{Q}_{m} & =\mathbf{B}+\mathbf{b} \boldsymbol{\eta}_{m} ; \quad \mathbf{b}=-\mathbf{B} \mathbf{1}
\end{aligned}
$$

where

$$
\begin{aligned}
& \boldsymbol{\eta}_{m}=\left(\frac{1}{c}\right)\left(\Delta_{m}^{0)}\left(n_{m 1}\right), \ldots, \Delta_{m}^{\left.\sigma_{m 1}-1\right)}\left(n_{m 1}\right), \ldots, \Delta_{m}^{0)}\left(n_{m q}\right), \ldots, \Delta_{m}^{\left.\sigma_{m q}-1\right)}\left(n_{m q}\right)\right) \\
& \left.\times\left(C_{m}^{1} \ldots C_{m}^{r}\right)^{\prime} \quad \text { Block product }\right)
\end{aligned}
$$


and

$$
\begin{aligned}
& \Delta_{m}^{0)}(n)=a^{*}(m-c n) \beta(n \mathbf{I}-\mathbf{B})^{-1} \\
& \mathbf{C}_{m}^{-1} \mathbf{a}=\left(C_{m}^{1} \ldots C_{m}^{r}\right)^{\prime}
\end{aligned}
$$

and $n_{m i}, i=1, \ldots, q$ are the complex roots (not necessarily distinct) with non-negative real parts of the $C$ - $L$ equation and $\Delta_{m}^{l)}(n)$ denotes the l'th derivative with respect to $n$ of $\Delta_{m}^{0)}(n)$.

Proof. Let us start considering the single Laplace transform in time of the ruin functions

$$
\begin{aligned}
& \psi_{m, u, y}^{*}=\int_{t=0}^{\infty} e^{-m t} \psi_{t, u, y} d t \\
& \Psi_{m, u, y}^{*}=\int_{t=0}^{\infty} e^{-m t} \Psi_{t, u, y} d t
\end{aligned}
$$

related of course via:

$$
\psi_{m, u, y}^{*}=m \Psi_{m, u, y}^{*}=\int_{t=0}^{\infty} m e^{-m t} \Psi_{t, u, y} d t
$$

The reader can easily realize that the expression above shows the multivariate ruin probability function for an exponentially killed process, see Avram and Usábel (2001) and Asmussen, Avram and Usábel (2002)

This function admits a simple renewal representation similar to the ultimate ruin probability

$$
\begin{aligned}
\psi_{m, u, y}^{*} & =\int_{t=0}^{\infty} m e^{-m t} \int_{0}^{t}(B(u+c s+y)-B(u+c s)) a(s) d s d t \\
& +\int_{t=0}^{\infty} m e^{-m t} \int_{0}^{t} \int_{0}^{u+c s} \Psi_{t-s, u+c s-z, y} b(z) a(s) d z d s d t \\
& =\int_{0}^{\infty}(B(u+c s+y)-B(u+c s))\left[e^{-m s} a(s)\right] d s \\
& +\int_{s=0}^{\infty} \int_{0}^{u+c s} \int_{t=s}^{\infty} m e^{-m(t-s)} \Psi_{t-s, u+c s-z, y} b(z)\left[e^{-m s} a(s)\right] d z d s d t \\
& =\int_{0}^{\infty}(B(u+c s+y)-B(u+c s)) a_{m}(s) d s \\
& +\int_{s=0}^{\infty} \int_{0}^{u+c s} \psi_{m, u+c s-z, y}^{*} b(z) a_{m}(s) d z d s
\end{aligned}
$$

in terms of a new shifted waiting times distribution with d.f. $a_{m}(t)=e^{-m t} a(t)$

$$
\psi_{m, u, y}^{*} \equiv \Psi_{\infty, u, y} \text { with waiting times d.f. } a_{m}(t)
$$


Let us now follow the reasoning presented in Asmussen (2000). Chapter 8 . Theorem 4.4. for the ultimate case when claim sizes are $\operatorname{PH}(\beta, \mathbf{B}, k)$

$$
\begin{aligned}
\psi_{m, u, \infty}^{*} & =\boldsymbol{\eta}_{m} e^{\mathbf{Q}_{m} u} \mathbf{1} \\
\mathbf{Q}_{m} & =\mathbf{B}+\mathbf{b} \boldsymbol{\eta}_{m} ; \quad \mathbf{b}=-\mathbf{B} \mathbf{1}
\end{aligned}
$$

where $\boldsymbol{\eta}_{m}$, the initial phase distribution of the ladder heights, is obtained in proposition 4.3 using a fixed point equation.

However, a different approach is used here based on proposition 4.1. a) in the mentioned text: it suffices to find the ladder height PH distribution of the shifted risk process (or killed following an exponential distribution)

$$
G_{m}(y)=\boldsymbol{\eta}_{m}\left(\mathbf{I}-e^{\mathbf{B} y}\right) \mathbf{1}
$$

Thence using lemma 3 (see below in this section)

$$
G_{m}(y)=\left(\frac{1}{c}\right) \mathbf{D}_{m, y} \mathbf{C}_{m}^{-1} \mathbf{a}
$$

It is easily proved that for $\operatorname{PH}(\beta, \mathbf{B}, k)$ claim sizes

$$
\begin{aligned}
B_{y}^{*}(n) & =\beta(n \mathbf{I}-\mathbf{B})^{-1}\left(\mathbf{I}-e^{\mathbf{B} y}\right) \mathbf{1} \\
D_{m, y}^{l}(n) & =a^{*}(m-c n) B_{y}^{*}(n) \\
& =\Delta_{m}^{l)}(n)\left(\mathbf{I}-e^{\mathbf{B} y}\right) \mathbf{1}
\end{aligned}
$$

and since $\mathbf{D}_{m, y}$ is a row vector (see (5)) and $\mathbf{C}_{m}^{-1} \mathbf{a}$ is a column vector with components $\left(C_{m}^{1} \cdots C_{m}^{r}\right)^{\prime}$ and the last two terms in the RHS above are independent of $n$, they may be factored outside the vector $\mathbf{D}_{m, y}$ using a block product in the first factor, denoted by $x$

$$
\begin{aligned}
& G_{m}(y)=\left(\frac{1}{c}\right) \mathbf{D}_{m, y} \mathbf{C}_{m}^{-1} \mathbf{a} \\
= & \frac{1}{c}\left(\Delta_{m}^{0)}\left(n_{m 1}\right), \ldots, \Delta_{m}^{\left.\sigma_{m 1}-1\right)}\left(n_{m 1}\right), \ldots, \Delta_{m}^{0)}\left(n_{m q}\right), \ldots, \Delta_{m}^{\left.\sigma_{m q}-1\right)}\left(n_{m 1}\right)\right) \\
& \times\left(C_{m}^{1} \cdots C_{m}^{r}\right)^{\prime}\left(\mathbf{I}-e^{\mathbf{B} y}\right) \mathbf{1}
\end{aligned}
$$

proving (11).

One should notice that the row vector $\boldsymbol{\eta}_{m} e^{\mathrm{Q}_{m} u}$ provides the initial phase structure when the process crosses the ruin barrier or reaches zero reserves (see again Asmussen (2000), chapter 8). Afterwards the claim causing ruin will evolve following the intensity matrix $B$ and obviously

$$
\psi_{m, u, y}^{*}=\boldsymbol{\eta}_{m} e^{\mathrm{Q}_{m} u}\left(\mathbf{I}-e^{\mathrm{B} y}\right) \mathbf{1},
$$

see also Avram and Usábel (2001) and Asmussen, Avram and Usábel (2001). Lemma 3 is now presented and proved. 
Lemma 3. The c.d.f. of the ladder height of the shifted (or exponentially killed) risk process

$$
G_{m}(y)=\left(\frac{1}{c}\right) \mathbf{D}_{m, y} \mathbf{C}_{m}^{-1} \mathbf{a}
$$

Proof. It is easy to see that

$$
G_{m}(y)=\psi_{m, 0, y}^{*}=\lim _{n \rightarrow \infty} n \psi_{m, n, y}^{* *}
$$

using the Tauberian theorems and

$$
\begin{aligned}
G_{m}(y) & =\lim _{n \rightarrow \infty} n \psi_{m, n, y}^{* *} \\
& =\lim _{n \rightarrow \infty} n \frac{B_{y}^{*}(n) a^{*}(m-c n)-\mathbf{D}_{m, y} \mathbf{C}_{m}^{-1} \mathbf{a}^{*}[m-c n]}{1-b^{*}(n) a^{*}(m-c n)} \\
& =-\mathbf{D}_{m, y} \mathbf{C}_{m}^{-1} \lim _{n \rightarrow \infty} n \mathbf{a}^{*}[m-c n]=\left(\frac{1}{c}\right) \mathbf{D}_{m, y} \mathbf{C}_{m}^{-1} \mathbf{a}
\end{aligned}
$$

because

$$
\lim _{n \rightarrow \infty} n \mathbf{a}^{*}[m-c n]=\lim _{n \rightarrow \infty} n((m-c n) \mathbf{I}-\mathbf{A})^{-1} \mathbf{a}=-\left(\frac{1}{c}\right) \mathbf{a}
$$

Remark 1. Corollary 4.1 in Asmussen (1992) can be also applied to obtain $\boldsymbol{\eta}_{m}$ using the negative (assumed distinct) roots of the Lundberg's equation. However the result is not proved to hold when multiple negative roots are found. Moreover, when the order of the claim sizes phase-type distribution is greater than the one modelling the waiting times, $k>r$, formula (14) is clearly more efficient because the number of roots to find is smaller.

The multivariate ultimate ruin probability can be given by a phase-type distribution without inversions

$$
\Psi_{\infty, u, y}=\psi_{0, u, y}^{*}=\boldsymbol{\eta}_{0} e^{\mathbf{Q}_{0} u}\left(\mathbf{I}-e^{\mathbf{B} y}\right) \mathbf{1}
$$

(see Asmussen and Rolski (1992) and Avram and Usábel (2001) for the classical case). Most standard Laplace transform inversion techniques can given by the general formula

$$
\Psi_{t, u, y} \simeq \sum_{i=0}^{M} W_{M, i}(t) \boldsymbol{\eta}_{m_{M, i}(t)} e^{\mathbf{Q}_{m_{M, i}(t)} u}\left(\mathbf{I}-e^{\mathbf{B} y}\right) \mathbf{1}
$$

so that the multivariate finite time ruin probability can be expressed very conveniently as a sum of phase-type distributions of order $k$.

Remark 2. In Asmussen (1992), $\boldsymbol{\eta}_{m}$ was also obtained by an iterative solution. When inverting Laplace transforms, the precision digits of the Laplace transform 
to invert is crucial in many methods due to the magnitude of the weights $\left(W_{M, i}(t)\right)$, see for instance Usábel (1999) for details in the Gaver-Stehfest method. For the reason just mentioned, relying on an iterative procedure to obtain $\boldsymbol{\eta}_{m_{M, i}(t)}$ may endanger the accuracy.

\section{Numerical IlluStration}

As a numerical illustration table IIB in Wikstad (1971) is reproduced below. The claim size distribution is $P H(\beta, \mathbf{B}, 3)$

$$
\begin{aligned}
& \beta=\left(\begin{array}{lll}
0.0039793 & 0.1078392 & 0.8881815
\end{array}\right) \\
& \mathbf{B}=\operatorname{diag}\left\{\begin{array}{lll}
-0.014631 & -0.190206 & -5.514588
\end{array}\right\}
\end{aligned}
$$

and the waiting times $P H(\alpha, \mathbf{A}, 2)$

$$
\alpha=\left(\begin{array}{ll}
0.25 & 0.75
\end{array}\right) \quad \mathbf{A}=\operatorname{diag}\left\{\begin{array}{ll}
-0.4 & -2
\end{array}\right\}
$$

Tables I-IV were obtained using (15) and the Gaver-Stehfest method of inverting the Laplace transform with order $M=8$ (see Usábel (1999)) where

$$
\begin{aligned}
& W_{M, i}(t)=\frac{\ln (2)}{t}(-1)^{i+M / 2} \sum_{j=[(i+1) / 2]}^{M i n(i, M / 2)} \frac{j^{(M / 2)}(2 j) !}{(M / 2-j) ! j !(j-1) !(i-j) !(2 j-i) !} \\
& m_{M, i}(t)=\frac{i \ln (2)}{t} \quad i=1, \ldots, M
\end{aligned}
$$

Original results by Wikstad (1971) in brackets. The reader can see the match of 4 significant digits in many figures. Later, calculations were performed using $M=12$ with a perfect match of 4 significant digits in all cases. $\theta$ is the relative safety loading considered.

TABLE I

$\Psi_{1, u, \infty}$ TABLE IIB WiKSTAD (1971)

\begin{tabular}{rcccc}
\hline \hline & $t=1$ & $u$ & & \\
& $\mathbf{0}$ & $\mathbf{1}$ & $\mathbf{1 0}$ & $\mathbf{1 0 0}$ \\
\hline $\mathbf{0 . 0 5}$ & $0.3301(0.3300)$ & $0.1187(0.1186)$ & $0.0276(0.0276)$ & $0.0013(0.0013)$ \\
$\mathbf{0 . 1 0}$ & $0.3221(0.3221)$ & $0.1180(0.1180)$ & $0.0275(0.0275)$ & $0.0013(0.0013)$ \\
$\theta \mathbf{0 . 1 5}$ & $0.3147(0.3146)$ & $0.1174(0.1173)$ & $0.0274(0.0274)$ & $0.0013(0.0013)$ \\
$\mathbf{0 . 2 0}$ & $0.3077(0.3076)$ & $0.1168(0.1167)$ & $0.0273(0.0273)$ & $0.0013(0.0013)$ \\
$\mathbf{0 . 2 5}$ & $0.3011(0.3010)$ & $0.1161(0.1161)$ & $0.0272(0.0272)$ & $0.0013(0.0013)$ \\
$\mathbf{0 . 3 0}$ & $0.2949(0.2948)$ & $0.1156(0.1155)$ & $0.0271(0.0271)$ & $0.0013(0.0013)$ \\
\hline \hline
\end{tabular}


TABLE II

$\Psi_{10, u, \infty}$ TABLE IIB WiKSTAD (1971)

\begin{tabular}{rcccc}
\hline \hline & \multirow{2}{*}{$t=10$} & $\mathbf{u}$ & & \\
& $\mathbf{0}$ & $\mathbf{1}$ & $\mathbf{1 0}$ & $\mathbf{1 0 0}$ \\
\hline $\mathbf{0 . 0 5}$ & $0.5960(0.5962)$ & $0.4344(0.4345)$ & $0.1686(0.1686)$ & $0.0103(0.0103)$ \\
$\mathbf{0 . 1 0}$ & $0.5843(0.5845)$ & $0.4259(0.4260)$ & $0.1647(0.1647)$ & $0.0103(0.0103)$ \\
$\theta \mathbf{0 . 1 5}$ & $0.5730(0.5731)$ & $0.4176(0.4178)$ & $0.1610(0.1610)$ & $0.0103(0.0103)$ \\
$\mathbf{0 . 2 0}$ & $0.5621(0.5622)$ & $0.4097(0.4098)$ & $0.1574(0.1574)$ & $0.0102(0.0102)$ \\
$\mathbf{0 . 2 5}$ & $0.5515(0.5517)$ & $0.4019(0.4021)$ & $0.1540(0.1539)$ & $0.0102(0.0102)$ \\
$\mathbf{0 . 3 0}$ & $0.5413(0.5415)$ & $0.3945(0.3946)$ & $0.1506(0.1506)$ & $0.0101(0.0101)$ \\
\hline \hline
\end{tabular}

TABLE III

$\Psi_{100, u, \infty}$ TABLE IIB WiKSTAD (1971)

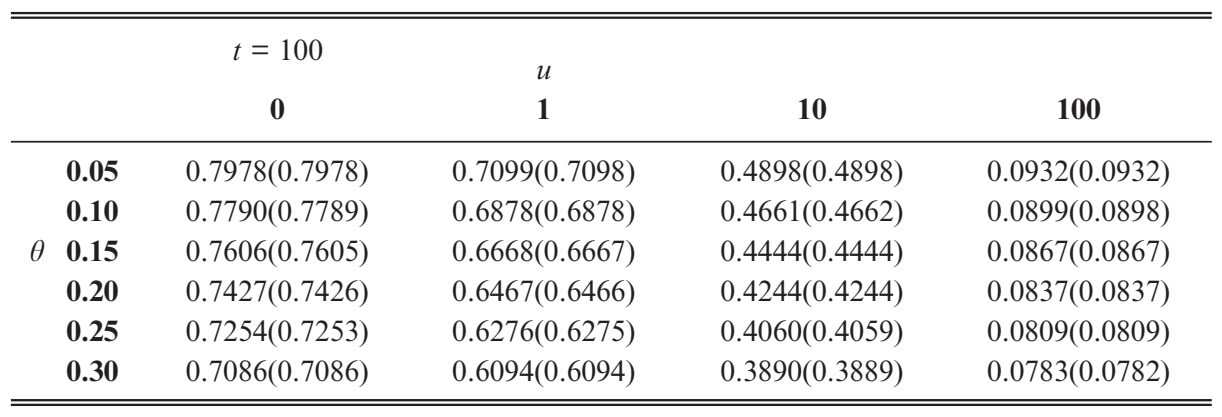

TABLE IV

$\Psi_{1000, u, \infty}$ TABLE IIB WIKSTAD (1971)

\begin{tabular}{ccccc}
\hline \hline & $t=1000$ & $u$ & & \\
& $\mathbf{0}$ & $\mathbf{1}$ & $\mathbf{1 0}$ & $\mathbf{1 0 0}$ \\
\hline $\mathbf{0 . 0 5}$ & $0.9113(0.9113)$ & $0.8724(0.8724)$ & $0.7690(0.7691)$ & $0.4207(0.4209)$ \\
$\mathbf{0 . 1 0}$ & $0.8890(0.8890)$ & $0.8429(0.8429)$ & $0.7247(0.7247)$ & $0.3708(0.3710)$ \\
$\theta \mathbf{0 . 1 5}$ & $0.8659(0.8659)$ & $0.8130(0.8130)$ & $0.6815(0.6815)$ & $0.3273(0.3274)$ \\
$\mathbf{0 . 2 0}$ & $0.8425(0.8425)$ & $0.7833(0.7833)$ & $0.6403(0.6403)$ & $0.2896(0.2897)$ \\
$\mathbf{0 . 2 5}$ & $0.8191(0.8191)$ & $0.7543(0.7543)$ & $0.6015(0.6015)$ & $0.2571(0.2572)$ \\
$\mathbf{0 . 3 0}$ & $0.7961(0.7961)$ & $0.7263(0.7263)$ & $0.5655(0.5655)$ & $0.2292(0.2293)$ \\
\hline \hline
\end{tabular}

Let us now use a different method of inversion of the Laplace transform and obtain Table 8 in Thorin \& Wikstad (1973), where the waiting times distribution follows (16) and the claim size distribution is $P H(\beta, \mathbf{B}, 5)$ 


\section{$\beta=\left(\begin{array}{lllll}0.6635948 & 0.3114878 & 0.02405664 & 0.0008425574 & 0.00001823254\end{array}\right)$ $\mathbf{B}=\operatorname{diag}\left\{\begin{array}{lllll}3.675472 & 0.7116063 & 0.09447445 & 0.00932298 & 0.000496562\end{array}\right\}$}

This distribution is considered a rough approximation to the Pareto distribution $B(x)=1-(1+2 x)^{-3 / 2}$.

Now the approximations for the finite time multivariate ruin probability can be then given by $\Psi_{t, u, y} \simeq S_{M}^{0}(t)$ obtained from (15) where

$$
\begin{aligned}
& m_{M, i}(t)=\frac{A}{2 t}+\frac{i \pi}{t} I, \quad I=\sqrt{-1}, \quad A=15 \ln (10), \quad i=0,1, \ldots \\
& W_{M, 0}(t)=\frac{e^{A / 2}}{2 t} ; \quad W_{M, i}(t)=(-1)^{i} \frac{e^{A / 2}}{t}, \quad i=1,2, \ldots
\end{aligned}
$$

where $A$ is a parameter controlling the maximum significant digits of the approximation. Later the error can be reduced and tight interval approximations found, as shown in Usábel (2001) using the acceleration technique

$$
S_{n}^{j}(t)=\frac{S_{n}^{j-1}(t)+S_{n+1}^{j-1}(t)}{2} n=1, \ldots, M \quad j=0, \ldots, M-n
$$

The interval estimations in tables V-VII were obtained using $S_{9}^{20}(t), S_{10}^{20}(t)$ and $S_{11}^{20}(t)$ so that the maximum $M$ considered was $M=31$, see Usábel (2001) for details

\begin{tabular}{|c|c|c|c|}
\hline & $\begin{array}{c}t=100 \\
\mathbf{0}\end{array}$ & $\begin{array}{c}\mathbf{u} \\
\mathbf{1 0 0}\end{array}$ & 1000 \\
\hline-0.10 & $(9.10914698,9.10914699) 10^{-1}$ & $(4.45651275,4.45651276) 10^{-2}$ & $(1.141111286,1.141111288) 10^{-3}$ \\
\hline-0.05 & $(8.92746514,8.92746515) 10^{-1}$ & $(4.24507550,4.24507551) 10^{-2}$ & $(1.138840749,1.138840750) 10^{-3}$ \\
\hline$\theta \quad \mathbf{0 . 0 0}$ & $(8.73894475,8.73894477) 10^{-1}$ & $(4.06047828,4.06047829) 10^{-2}$ & $(1.136641913,1.136641915) 10^{-3}$ \\
\hline 0.05 & $(8.54651224,8.54651225) 10^{-1}$ & $(3.897856107,3.897856112) 10^{-2}$ & $(1.134508219,1.134508221) 10^{-3}$ \\
\hline 0.10 & $(8.35266374,8.35266375) 10^{-1}$ & $(3.753304129,3.753304134) 10^{-2}$ & $(1.132433562,1.132433563) 10^{-3}$ \\
\hline
\end{tabular}

TABLE V

$\Psi_{100, u, \infty}$ TABLE 8 THORIN \& WiKSTAD (1971)

TABLE VI

$\Psi_{1000, u, \infty}$ TABLE 8 THORIN \& WiKSTAD (1971)

\begin{tabular}{rrccc}
\hline \hline & \multicolumn{1}{c}{$t=1000$} & $\mathbf{u}$ & \\
& & $\mathbf{0}$ & $\mathbf{1 0 0}$ & $\mathbf{1 0 0 0}$ \\
\hline $\mathbf{- 0 . 1 0}$ & $(9.77349281,9.77349283) 10^{-1}$ & $4.55579375,4.55579376) 10^{-1}$ & $(1.251313553,1.251313554) 10^{-2}$ \\
$\mathbf{- 0 . 0 5}$ & $(9.60855502,9.60855504) 10^{-1}$ & $(3.65024541,3.65024542) 10^{-1}$ & $(1.209846323,1.209846325) 10^{-2}$ \\
$\theta$ & $\mathbf{0 . 0 0}$ & $(9.40788891,9.40788892) 10^{-1}$ & $(2.971960518,2.971960525) 10^{-1}$ & $(1.175856267,1.175856269) 10^{-2}$ \\
& $\mathbf{0 . 0 5}$ & $(9.18570432,9.18570433) 10^{-1}$ & $(2.46971941,2.46971942) 10^{-1}$ & $(1.147302851,1.147302852) 10^{-2}$ \\
& $\mathbf{0 . 1 0}$ & $(8.95361315,8.95361316) 10^{-1}$ & $(2.092929693,2.092929698) 10^{-1}$ & $(1.122718227,1.122718229) 10^{-2}$ \\
\hline \hline
\end{tabular}


TABLE VII

$\Psi_{10000, u, \infty}$ TABLE 8 THORIN \& WIKSTAD (1971)

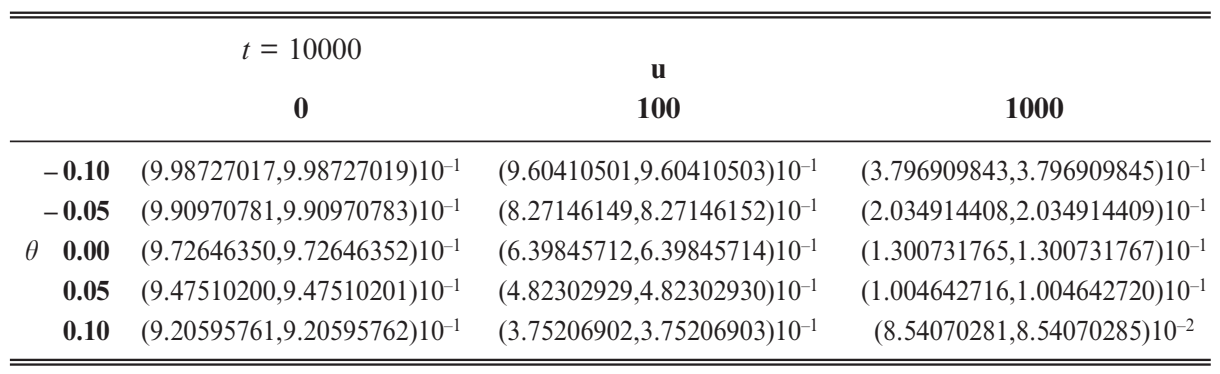

Finally, in table VIII, the deficit at ruin is also considered, $y=6$, and the interval estimations were obtained with $S_{4}^{14}(t), S_{5}^{14}(t)$ and $S_{6}^{14}(t)$ so that the maximum $M=20$.

TABLE VIII

$\Psi_{100, u, 6}$ TABLE 8 THORIN \& WiKSTAD (1971)

\begin{tabular}{|c|c|c|c|c|}
\hline & & $\begin{array}{c}t=100 \\
\mathbf{0}\end{array}$ & $\underset{100}{\mathrm{u}}$ & 1000 \\
\hline \multirow{5}{*}{$\theta$} & -0.10 & $(8.0586,8.0595) 10^{-1}$ & $(7.272,7.273) 10^{-3}$ & $(8.923,8.924) 10^{-6}$ \\
\hline & -0.05 & $(7.848,7.849) 10^{-1}$ & $(6.3738,6.3744) 10^{-3}$ & $(8.472,8.473) 10^{-6}$ \\
\hline & 0.00 & $(7.638,7.639) 10^{-1}$ & $(5.6405,5.6411) 10^{-3}$ & $(8.072,8.073) 10^{-6}$ \\
\hline & 0.05 & $(7.431,7.432) 10^{-1}$ & $(5.038,5.039) 10^{-3}$ & $(7.718,7.719) 10^{-6}$ \\
\hline & 0.10 & $(7.228,7.229) 10^{-1}$ & $(4.540,4.541) 10^{-3}$ & $(7.404,7.405) 10^{-6}$ \\
\hline
\end{tabular}

The reader can easily see that the incorporation of the deficit to the calculations is trivial using (15). All calculations were performed in Maple V with 22 significant digits.

\section{CONCLUding COMMENTS}

From the theoretical point of view, this paper offers

1. Theorem 1. A generalization of formula 3.4 in Thorin (1971) to phase-type waiting times, instead of just hyperexponential, and considering an arbitrary claim size distribution. The deficit at ruin is also introduced. The phase-type environment is clearly much more powerful that the hyperexponential case considered in the works by Cramer and Thorin (see the introduction for details).

2. Theorem 2. An alternative representation of the crucial row vector $\boldsymbol{\eta}_{m}$, the initial phase structure of the ladder heights of the risk process in a phase-type 
environment (which yields then immediately the killed multivariate ruin probabilities by a mere matrix exponentiation). The new result uses the complex roots with non-negative real part of the CL's equation instead of the negative (assumed distinct) ones in Asmussen (1992).

From the practical point of view, the purpose of this work is showing how finite time multivariate ruin probabilities, in a phase-type environment, can be approximated efficiently with only one Laplace transform inversion. Moreover, the mentioned ruin probability can be given by a sum of phase-type distributions of the same order as the one modelling the claim size, $k$ (see (15)).

In actuarial practice, clearly, only empirical distributions will be available for the claim size and waiting times of the risk process. The phase-type, as mentioned earlier, is a dense family and can approximate any distribution. The problem of fitting phase-type distribution to empirical data is a most interesting issue in applied probability, unfortunately beyond the scope of this work. The reader is referred, for instance, to the works by Bux \& Herzog (1977), Johnson \& Taaffe (1985), Lang \& Arthur (1994) or Mitchell \& van de Liefvoort (2000) for methods, illustrations and software available. For an actuarial application see also Tseggai (2000).

When approximating empirical distributions using phase-type families, the claim size distribution is more likely to be complicated due to possible long tail behaviours. This translates into a higher order phase-type representation for the claim size and hence in a larger number of negative roots $(k \gg r)$. Therefore, we find the representation of $\boldsymbol{\eta}_{m}$ using the $r$ non-negative roots of the Lundberg's equation instead of the $k$ non-positive ones quite useful for computations.

The phase-type environment, besides of practical interest due to the simplicity of the results, can offer many valuable generalizations to the risk theory modelling such as the embedding of the distribution in a semi-markovian environment, see chapter 8 in Asmussen (2000) or Avram, Pistorius and Usábel (2003)

\section{REFERENCES}

Asmussen, S. (1984) Approximations for the probability of ruin within finite time. Scandinavian Actuarial Journal, 31-57.

Asmussen, S. (1987) Applied Probability and Queues. Wiley, New York.

Asmussen, S. and RolSKI (1991) Computational Methods in risk theory: a matrix-algorithmic approach. Insurance: Mathematics and Economics, 10, 259-274.

Asmussen, S. (1992) Phase-type representations in random walk and queueing problems. The Annals of Probability, 20(2), 772-789

Asmussen, S. and BLADT, M. (1996) Renewal theory and queueing algorithms for matrix-exponential distributions. Matrix-Analytic Methods in Stochastic Models (A.S Alfa \& S. Chkravarty, eds.), 313-341

Asmussen, S. (2000). Ruin Probabilities. World Scientific.

Asmussen, S. and HojgaArd, B. (1999) Approximations for finite time ruin probabilities in the renewal model. Scandinavian Actuarial Journal, 99, 106-119.

Asmussen, S., Avram, F. and Usábel, M. (2002). Erlangian approximations for finite-horizon ruin probabilities. ASTIN Bulletin 32.

Avram, F. and UsÁbel, M. (2001) Finite time ruin probabilities with one Laplace inversion. To appear in Insurance: Mathematics and Economics. 
Avram, F., Pistorius, M. and Usábel, M. (2003) The two barriers ruin problem via a WienerHöpf decomposition approach. Annals of the University of Craiova, Math. Comp. Sci. Series, 30, $1-9$.

Bohman, H. (1971) Ruin probabilities. Skandinavisk Aktuarietidskrift, 159-163.

Bohman, H. (1974) Fourier Inversion-Distribution functions-Long tails. Scand. Actuarial Journal, $43-45$

Bohman, H. (1975) Numerical inversion of characteristic functions. Scan. Act. Journal, 121-124

Bux, W. and Herzog, U. (1977). The Phase Concept: Approximation of Measured Data and Performance Analysis. In: K.M. Chandy and M. Reiser. eds., Computer Performance. NorthHolland, Amsterdam, 23-38.

Cramèr, H. (1955) Collective Risk Theory. Jubille Volume of F. Skandia.

Dickson, D. and Egidio Dos Reis, A. (1996) On the distribution of the duration of negative surplus. Scandinavian Actuarial Journal, 148-164.

EGIDIO DOs ReIs, A. (1993). How long is the surplus below zero? Insurance: Mathematics and Economics, 12, 23-38.

EGIDIO DOS REIS, A. (2000). On the moments of ruin and recovery times. Insurance: Mathematics and Economics, 27, 331-343.

Gerber, H. , Goovaerts, M. and KaAs, R. (1987) On the probability and severity of ruin. ASTIN Bulletin 17, 151-163.

Johnson, M.A. and TaAffe, M.R. (1985) Matching Moments to Phase Distributions: Mixture of Erlang distributions of common order. Stochastic Models 6, 259-281.

Lang, A and Arthur, J.L. (1994) Parameter Approximation for Phase type Distributions. Technical Report, Oregon Sate University, Corvallis.

Mitchell, K. and van De Liefvoort, A. (2000) Constructing a Matrix Exponential Distribution Using Moment Matching, In Approximation Models of Feed-Forward G/G/1/N Queuing Networks with Correlated Arrivals. Computer Science Telecommunications Program 5100 Rockill, Kansas City.

Neuts, M. (1975) Probability distibutions of phase-type. Liber Amicorum Professor Emeritus H. Florin, Dep. of Mathematics, University of Louvain, Belgium, 173-206.

Neuts, M. (1977) Algorithms for the waiting time distribution under various queue disciplines in the M/G/1 queue with service time distribution of phase-type. TIMS Studies in the Management Science, 7, 177-197

PiessENs, R. (1969) New quadrature formulas for the numerical inversion of Laplace transforms. BIT 9, 351-361

Rolski, T., Schmidli, H., Schmidt, V. and Teugels, J. (1999) Stochastic Processes for Insurance and Finance. Wiley, Chichester.

SEAL, H. (1971) Numerical calculation of the Bohman-Esscher family of convolution-mixed negative binomial distribution functions. Mitt. Verein. schweiz. Versich.-Mathr. 71, 71-94.

SEAL, H. (1974) The numerical calculation of $U(w, t)$, the probability of non-ruin in an interval (0,t). Scan. Act. Journal, 121-139.

Seal, H. (1977) Numerical inversion of characteristic functions. Scan. Act. Journal, 48-53.

STANFORD, D. and STROINSKI, K. (1994) Recursive methods for computing finite-time ruin probabilities for phase-type distributed claim sizes. Astin Bulletin 24(2), 235-254.

Stanford, D., Stroinski, K. and Lee, K. (2000) Ruin probabilities based at claim instants for some non-Poisson claim processes. Insurance: Mathematics and Economics, 26, 251-267.

THORIN, O. (1970) Some remarks on the ruin problem in case the epochs of claims form a renewal process. Skandinavisk Aktuarietidskrift, 29-50.

THorin, O. (1971) Further remarks on the ruin problem in case the epochs of claims form a renewal process. Skandinavisk Aktuarietidskrift, 14-38, 121-142.

THORIN, O. (1973) The ruin problem in case the tail of a distribution is completely monotone. Skandinavisk Aktuarietidskrift, 100-119.

THORIN, O. (1977) Ruin probabilities prepared for numerical calculation. Scandinavian Actuarial Journal.

Thorin, O. (1982) Probabilities of Ruin. Scandinavian Actuarial Journal, 65-102.

THORIN, O. and WiKSTAD, N. (1973) Numerical evaluation of ruin probabilities for a finite period. ASTIN Bulletin VII:2, 138-153. 
TsegGai, A. (2000) General Solutions of ultimate ruin probabilities. Master in Actuarial Science Thesis. Heriot-Watt University.

UsÁBEL, M. (1999) Calculating multivariate ruin probabilities via Gaver-Syehfest inversion technique. Insurance: Mathematics and Economics, 25, 133-142.

UsÁBEL, M. (2001) Ultimate ruin probabilities for generalized gamma convolutions claim sizes. ASTIN Bulletin, 31(1), 63-83.

WiKSTAD, N. (1971) Exemplifications of ruin probabilities. ASTIN Bulletin, VI, part 2.

WiKSTAD, N. (1977) How to calculate Ruin probabilities according to the classical Risk Theory. Scand. Actuarial Journal.

WiLLmot, G. (1998) On a class of approximations for ruin and waiting time probabilities. Oper. Res. Lett. 22, 27-32

WiLlmot, G. (1999) A Laplace transform representation in a class of renewal queueing and risk process. J. Appl. Prob. 36, 570-584.

Willmot, G. (2000) On the evaluation of the conditional distribution of the deficit at the ruin time. Scandinavian Actuarial Journal, 100(1) 63-79.

\section{ApPendix A}

Lemma 4. When the waiting times of the risk process (1) follows a PH( $\alpha, \mathbf{A}, r)$ distribution with d.f. $a(t)=\alpha e^{\mathrm{A} t} \mathbf{a}$ and Laplace transform $a^{*}(n)=\alpha \mathbf{a}^{*}[n]$ where

$$
\begin{aligned}
\mathbf{a}^{*}[m-c n] & =((m-c n) \mathbf{I}-\mathbf{A})^{-1} \mathbf{a} \\
\mathbf{a} & =-\mathbf{A} \mathbf{1}
\end{aligned}
$$

then

a)

$$
\begin{aligned}
& \int_{0}^{\infty} e^{-m t} \int_{0}^{\infty} e^{-n u} \int_{s=0}^{t} \int_{z=0}^{u+c s} \psi_{t-s, u+c s-z, y} b(z) a(s) d z d s d u d t \\
& =\psi_{m, n, y}^{* *} b^{*}(n) a^{*}(m-c n)-\mathbf{G}_{y}(m) \mathbf{a}^{*}[m-c n]
\end{aligned}
$$

where

$$
\mathbf{G}_{y}(m)=\alpha \psi_{m, \frac{m \mathbf{I}-\mathbf{A}}{c}, y}^{* *} b^{*}\left(\frac{m \mathbf{I}-\mathbf{A}}{c}\right)
$$

b)

$$
\begin{aligned}
& \int_{0}^{\infty} e^{-m t} \int_{0}^{\infty} e^{-n u}(B(u+c t+y)-B(u+c t)) a(t) d u d t \\
& =a^{*}(m-c n) B_{y}^{*}(n)-\mathbf{H}_{y}(m) \mathbf{a}^{*}[m-c n]
\end{aligned}
$$

where

$$
\begin{aligned}
B_{y}^{*}(n) & =\int_{a=0}^{\infty}(B(a+y)-B(a)) e^{-n a} d a \\
\mathbf{H}_{y}(m) & =\alpha \int_{a=0}^{\infty}(B(a+y)-B(a)) e^{-(m \mathbf{I}-\mathbf{A}) \frac{a}{c}} d a \\
& =\alpha B_{y}^{*}\left(\frac{m \mathbf{I}-\mathbf{A}}{c}\right)
\end{aligned}
$$


Proof. a) Using the definition of the double-Laplace transform

$$
\int_{t=0}^{\infty} \int_{u=0}^{\infty}\left(\int_{s=0}^{t} \int_{z=0}^{u+c s} \psi_{t-s, u+c s-z, y} b(z) a(s) d z d s\right) e^{-m t-n u} d u d t
$$

we find after the change of variables $t-s=w, \quad u+c s-z=v, \quad s=s, \quad z=z$ that it equals:

$$
\int_{w=0}^{\infty} \int_{v=0}^{\infty} \psi_{w, v, y} e^{-m w-n v} \int_{z=0}^{\infty} b(z) e^{-n z} \int_{s=0}^{(v+z) / c} a(s) e^{-(m-c n) s} d s d z d v d w
$$

Under the phase-type assumption, the inner integral is

$$
\begin{aligned}
& \int_{s=0}^{(v+z) / c} a(s) e^{-(m-c n) s} d s=\alpha \int_{s=0}^{(v+z) / c} e^{\mathbf{A} s} e^{-(m-c n) s} d s \mathbf{a} \\
& =\alpha\left(\mathbf{I}-e^{-\frac{v+z}{c}((m-c n) \mathbf{I}-\mathbf{A})}\right)((m-c n) \mathbf{I}-\mathbf{A})^{-1} \mathbf{a}
\end{aligned}
$$

substituting into (18) we obtain the result.

$$
\psi_{m, n, y}^{* *} b^{*}(n) a^{*}(m-c n)-\mathbf{G}_{y}(m) \mathbf{a}^{*}[m-c n]
$$

where the row vector $\mathbf{G}(m)$ is given by

$$
\begin{aligned}
\mathbf{G}_{y}(m) & =\alpha \int_{w=0}^{\infty} \int_{v=0}^{\infty} \psi_{w, v, y} e^{-m w} e^{-(m \mathbf{I}-\mathbf{A}) \frac{v}{c}} \int_{z=0}^{\infty} b(z) e^{-(m \mathbf{I}-\mathbf{A}) \frac{v}{c}} d z d v d w \\
& =\alpha \psi_{m, \frac{m \mathbf{I}-\mathbf{A}}{c}, y}^{* *} b^{*}\left(\frac{m \mathbf{I}-\mathbf{A}}{c}\right)
\end{aligned}
$$

b) For $a(t)=\alpha e^{\mathbf{A t}} \mathbf{a}$ we can obtain the double-Laplace transform

$$
\int_{0}^{\infty} \int_{0}^{\infty}(B(u+c t+y)-B(u+c t)) \alpha e^{\mathbf{A} t} \mathbf{a} e^{-m t-n u} d u d t
$$

After the change of variables

$$
u+c t=a \quad t=t
$$

and shifting the order of integration we easily get

$$
\begin{aligned}
& \int_{a=0}^{\infty}(B(a+y)-B(a)) e^{-n a} \int_{t=0}^{(a / c)} \alpha e^{-((m-c n) \mathbf{I}-\mathbf{A}) t} \mathbf{a} d t d a \\
& =\alpha \int_{a=0}^{\infty}(B(a+y)-B(a)) e^{-n a}\left(\mathbf{I}-e^{-((m-c n) \mathbf{I}-\mathbf{A}) \frac{a}{c}}\right) d a((m-c n) \mathbf{I}-\mathbf{A})^{-1} \mathbf{a}
\end{aligned}
$$

and obtain the result

$$
a^{*}(m-c n) B_{y}^{*}(n)-\mathbf{H}_{y}(m) \mathbf{a}^{*}[m-c n]
$$




\section{ApPendix B: Phase-type Distributions}

Definition 1. A phase-type distribution $B(x), P H(\beta, \mathrm{B}, k)$ henceforth, is the distribution of the absorbtion time $\xi$ of a finite (transient) Markov process $J_{t}$ on a state space $\{C, 1, \ldots, k\}$ with $k+1$ states to an absorbing state $C$ called cemetery. Here, $\boldsymbol{B}$ denotes the restriction of the intensity matrix of $J$ to the transient states $\{1, \ldots, k\}, \beta$ denotes the initial distribution (written as a row vector) of $J_{0}$ on $\{1$, $\ldots, k\}$, and $\xi=\inf \left\{t>0: J_{t}=C\right\}$

It follows that

$$
\begin{aligned}
& B(x)=\mathbb{P}_{\beta}[\xi \leq x]=1-\beta e^{\mathbf{B} x} \mathbf{1} \\
& b(x)=\beta e^{\mathbf{B} x} \boldsymbol{b}
\end{aligned}
$$

where $\mathbf{1}=(1 \ldots 1)^{\prime}, \boldsymbol{b}=-\boldsymbol{B} \mathbf{1}$, and the Laplace transform of the density is:

$$
\begin{aligned}
b^{*}(s) & =\beta(s \mathbf{I}-\mathbf{B})^{-1} \mathbf{b} \\
& =\beta \boldsymbol{b}^{*}[s] \quad \text { (see item } 2 \text { below) }
\end{aligned}
$$

\section{Notes:}

1) The vector $\boldsymbol{b}$ yields the rates of absorbtion from the various phases.

2) The column vector of Laplace transforms $\mathbf{b}^{*}[s]=\left(b_{i}^{*}(s), i=1, \ldots, k\right)$, where $b_{i}^{*}(s)=\mathbb{E}_{i} e^{-s \xi}$ denotes the Laplace transform of the absorbtion time starting in phase $i$, given by

$$
\mathbf{b}^{*}[s]=(s \mathbf{I}-\mathbf{B})^{-1} \mathbf{b}
$$

3) The phase-type family is dense (in the sense that any probability distribution can be approximated using a phase-type distribution) and hence of considerable practical interest.

4) The phase-type distributions have been used frequently in the recent actuarial and queueing literature. The reader is referred to the works by Neuts (1975, 1977), Asmussen (1987, 92), Asmussen and Rolski (1991) and Asmussen and Bladt (1996) for further properties and applications.

\section{F. Avram}

Dept. of Mathematics

Université de Pau

France

Floris.Avram@univ-pau.fr

M. USÁBEL

Dept. of Business Adm.

Universidad Carlos III de Madrid

Spain

usabel@emp.uc3m.es 